\title{
Model-based determination of dielectric function by STEM low-loss EELS
}

\author{
Liang Zhang* and Stuart Turner \\ EMAT, University of Antwerp, Groenenborgerlaan 171, 2020 Antwerpen, Belgium \\ Fons Brosens \\ Physics Department, University of Antwerp, Groenenborgerlaan 171, 2020 Antwerpen, Belgium \\ Jo Verbeeck \\ EMAT, University of Antwerp, Groenenborgerlaan 171, 2020 Antwerpen, Belgium
}

(Received 5 October 2009; revised manuscript received 23 November 2009; published 8 January 2010)

\begin{abstract}
Dielectric properties of materials are crucial in describing the electromagnetic response of materials. As devices are becoming considerably smaller than the optical wavelength, the conventional measuring methods based on optical response are limited by their spatial resolution. Electron energy loss spectroscopy performed in a scanning transmission electron microscope is a good alternative to obtain the dielectric properties with excellent spatial resolution. Due to the overlap of diffraction discs in scanning transmission electron microscopy, it is difficult to apply conventional experimental settings to suppress retardation losses. In this contribution, a relativistic dielectric model for the loss function is presented which is used in a model based optimization scheme to estimate the complex dielectric function of a material. The method is applied to experiments on bulk diamond and $\mathrm{SrTiO}_{3}$ and shows a good agreement with optical reference data when retardation effects are included. Application of this technique to nanoparticles is possible but several theoretical assumptions made in the model of the loss function are violated and interpretation becomes problematic.
\end{abstract}

DOI: 10.1103/PhysRevB.81.035102 PACS number(s): 79.20.Uv, 52.25.Mq, 04.25.-g, 68.37.Ma

\section{INTRODUCTION}

Dielectric properties of materials describe the response of materials to electromagnetic radiation ${ }^{1}$ and as such form the basis for many electronic and optical applications such as fiber optics, gate oxides, photonic crystals, and semiconductor devices. ${ }^{1-3}$ Typically this response is measured by using electromagnetic radiation such as light in the appropriate wavelength range for a given application and characterized by a refractive index $n$ and an absorption coefficient $\kappa$. Alternatively the response can be written as a complex dielectric constant $\varepsilon=\varepsilon_{1}+i \varepsilon_{2}$, where $\varepsilon_{1}=n^{2}-\kappa^{2}$ and $\varepsilon_{2}=2 n \kappa$.

With the ever decreasing size of devices, there is a need to investigate this dielectric response on scales which are smaller or comparable to optical wavelengths. Electron beams are good candidates for probing the local electronic response with nanometer spatial resolution. Low-loss electron energy loss spectroscopy (EELS) in an electron microscope would be a good candidate for this task, but it remains to be discussed how the obtained energy loss function can be linked to the optical response on small scales.

In the past, several researchers have shown that under certain conditions (bulk isotropic materials, low angle scattering, nonrelativistic electrons) the electron energy loss function is identical to the photon loss function and can be Kramers Kronig transformed into a complex dielectric function. ${ }^{4-6}$ Although this looks very promising, the data treatment turns out to be problematic and several issues arise. First of all the zero loss peak has to be subtracted which is difficult since no good analytical function exist that can fully describe the experimental shape of this peak. Even recording an experimental zero loss peak does usually not work due to different scattering properties and the resulting aberration effects in the spectrometer. ${ }^{7}$ Secondly a deconvo- lution step is needed to remove multiple scattering effects which also introduces artifacts. ${ }^{8}$ On top of these data treatment issues, more fundamental problems arise because of the relativistic nature of the electrons which can cause retardation effects in certain materials. ${ }^{9,10}$ Also the shape of the sample becomes important and surface effects arise while the sample starts to deviate from a true bulk sample. Retardation and shape effects have been theoretically described $9,11,12$ and some attempts have been made to apply these descriptions to experimental data. ${ }^{13}$ It was shown that retardation effects were crucial in determining the band gap from EELS spectra. ${ }^{13}$

It should be noted that in conventional transmission electron microscope (TEM) setups, these retardation effects can be suppressed ${ }^{4,5,13}$ by avoiding scattering angles where these effects are strong or by lowering the acceleration voltage. ${ }^{14}$ For focused probes, however, angle selection does not work since the diffraction pattern consists of convergent beam electron diffraction (CBED) discs. On the other hand, it is exactly the focused probes that we need for probing the dielectric response with high spatial resolution and therefore another way of treating the retardation effects is needed. Iterative schemes were shown to be promising for obtaining the complex dielectric function by taking into account the theoretical description of the loss function including retardation and surface effects. ${ }^{6}$

In this paper we will describe first attempts to obtain the complex dielectric function using a model based approach. The model consists of a parametric piecewise linear description of $\operatorname{Im}(-1 / \varepsilon)$ which can be analytically Kramers Kronig (KK) transformed to $\operatorname{Re}(1 / \varepsilon)$. Making use of the formula for the scattering probability, taking into account relativistic effects, we obtain a model for the loss function which can be iteratively updated to obtain a good fit with the experimental 
spectrum. The advantage of this method is that it works on a single experimental spectrum and no complicated experimental settings are needed. The method is equally valid for spectra obtained in STEM or TEM which makes it promising for use on high spatial resolution experiments. We first demonstrate the approach on two bulklike specimens (diamond and $\mathrm{SrTiO}_{3}$ ) and compare the results with optical data. For diamond the retardation effects are strong and therefore it is an excellent case study showing the need of including relativistic corrections. As a second example we show results on diamond nanoparticles where size effects start to play an important role.

\section{THEORY}

A model describing a low-loss EELS experiment can be constructed from three components: (i) a zero loss peak component; (ii) a single scattering distribution (SSD) describing the loss probability based on a parameterized complex dielectric function; and (iii) multiple scattering described by Fourier exponential convolution.

Each component can be parameterized and the parameters can be estimated by fitting the model to an experimentally obtained low-loss spectrum. The estimated parameters can then be interpreted as dielectric properties of the material depending on the way the model SSD is constructed.

\section{A. Zero loss peak}

When incident electrons bombard the sample, most of them are elastically scattered and they make up the zero loss peak (ZLP). Ideally this ZLP should be a delta function, but the finite energy distribution of the gun, electronic instabilities, aberrations in the spectrometer and quasielastic phonon scattering in the sample generally lead to a complicated asymmetric shape which is difficult to model. On the other hand, the tails of the ZLP at low-energy losses can easily be more intense than the inelastic scattering in that region which shows the importance of an accurate model of the ZLP. Two options are available for a model of the ZLP. Either an analytical function is taken which describes the complicated shape in all its details, or an experimentally obtained ZLP is used. The latter is seemingly the most promising technique, but unfortunately the shape of the ZLP is different with or without a sample due to the occurrence of different scattering angles leading to different aberrations in the spectrometer and due to the quasielastic phonon contributions. Alternatively one could also try to remove the ZLP from the experimental data by fitting an analytical function in a small fitting region where one assumes that the SSD is zero. In practice this approach seems to work best although it goes against the model based philosophy of avoiding all data treatment on the experimental data and it poses certain risks of subtracting parts of the spectra which actually belong to the SSD. It can be hoped that future developments in reducing spectrometer aberrations will make the experimentally obtained ZLP a better alternative.

\section{B. Multiple scattering}

To describe the effect of multiple scattering we use Fourier exponential convolution, ${ }^{7}$

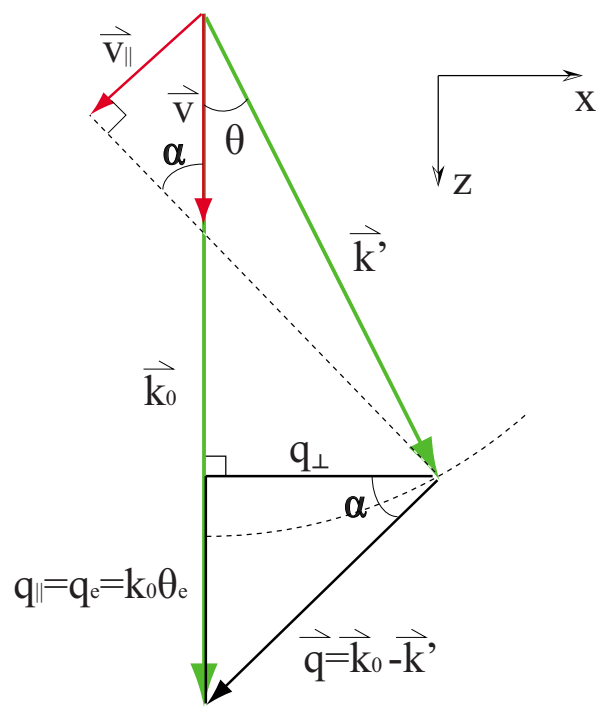

FIG. 1. (Color online) Scattering geometry of electrons scattered by a thin slab. $Z$ is the incident direction of the electron beam.

$$
J(E)=Z(E) \otimes \mathcal{F}^{-1}\left\{e^{-t} e^{t \mathcal{F}[S(E)]}\right\} .
$$

With $Z(E)$ the ZLP and $S(E)$ the SSD normalized to 1 and $t$ the relative thickness of the sample (compared to the inelastic mean-free path) which controls how much multiple scattering occurs. In case the ZLP is subtracted from the experiment, we are forced to do a deconvolution on the experiment rather than a convolution on the model. This constitutes another disadvantage of not having a good model for the ZLP. This disadvantage will likely be solved with improved spectrometer design where aberration effects become negligible in the future.

\section{Model for the SSD}

Inelastic scattering in the low-loss region is conveniently described in the dielectric formalism. ${ }^{7}$ A sketch of the scattering geometry is given in Fig. 1. A formula for the loss probability of a bulk material including retardation effects is given by De Abajo, ${ }^{12}$

$$
\begin{aligned}
\frac{\partial^{3} P^{l}}{\partial^{2} q_{\perp} \partial \omega}= & \frac{1}{(2 \pi)^{3} \hbar \omega} \frac{4 \pi e_{0}^{2} \omega}{v^{2} k_{0}^{2}} \operatorname{Im}\left\{\frac{1}{q^{2}}\left[\frac{1}{\varepsilon_{t r}(q, \omega)}-\frac{1}{\varepsilon_{l}(q, \omega)}\right]\right. \\
& \left.+\frac{\gamma^{2}}{\varepsilon_{t r}(q, \omega)\left(q_{\perp}^{2}+\gamma^{2} q_{E}^{2}\right)}\right\}
\end{aligned}
$$

With $\varepsilon_{t r}$ and $\varepsilon_{l}$ the transverse and longitudinal dielectric response. Note that the relativistic correction affects only the $q_{E}$ momentum transfer in the direction of the beam as shown in Fig. 1. The correction includes the difference of speed in the medium which is included in $\gamma=\sqrt{1-\varepsilon(0) \beta^{2}}$, with $\beta$ $=v / c$ and $\varepsilon(0)$ is the dielectric constant of the media. Assuming that $\varepsilon=\varepsilon_{t r}=\varepsilon_{l}$ in an isotropic material in the range of the measurements we get 


$$
\frac{\partial^{3} P^{l}}{\partial^{2} q_{\perp} \partial \omega}=\frac{1}{(2 \pi)^{3} \hbar \omega} \frac{4 \pi e_{0}^{2} \omega}{v^{2} k_{0}^{2}} \operatorname{Im}\left[\frac{\gamma^{2}}{\varepsilon(q, \omega)\left(q_{\perp}^{2}+\gamma^{2} q_{E}^{2}\right)}\right],
$$

For very small scattering angles and convergent angle equal to zero, we can neglect the dependence of the dielectric function on $\mathrm{q}$ and solve the collection angle integral analytically to get the SSD,

$$
\begin{aligned}
S(E)_{p} & =\frac{e_{0}^{2}}{v^{2} \pi^{2} \hbar^{2}} \operatorname{Im}\left[\int_{0}^{\theta_{C}} 2 \pi \theta d \theta \frac{\gamma^{2}}{\varepsilon(E)\left(\theta^{2}+\gamma^{2} \theta_{E}^{2}\right)}\right] \\
& =\frac{e_{0}^{2}}{v^{2} \pi \hbar^{2}} \operatorname{Im}\left[\frac{\gamma^{2}}{\varepsilon(E)} \ln \left(\frac{\theta_{C}^{2}+\gamma^{2} \theta_{E}^{2}}{\gamma^{2} \theta_{E}^{2}}\right)\right],
\end{aligned}
$$

When using convergent beam, Eq. (4) can be rewritten as

$$
S(E)_{c}=\frac{e_{0}^{2}}{v^{2} \pi^{2} \hbar^{2}} \operatorname{Im}\left[\int_{0}^{\theta_{C}+\alpha_{0}} 2 \pi \theta d \theta \frac{F\left(\alpha_{0}, \theta_{c}\right) \gamma^{2}}{\varepsilon(E)\left(\theta^{2}+\gamma^{2} \theta_{E}^{2}\right)}\right],
$$

which can be intergraded numerically with $\alpha_{0}$ is the convergent angle and $F\left(\alpha_{0}, \theta_{c}\right)$ is the normalized cross correlation. ${ }^{15}$

In a next step we chose a piecewise linear function to represent $\operatorname{Im}\left(\frac{-1}{\varepsilon(E)}\right)$,

$$
\operatorname{Im}\left(\frac{-1}{\varepsilon(E)}\right)=\alpha \sum_{i}\left(a_{i} E+b_{i}\right) H\left(E-E_{i}\right) H\left(E_{i+1}-E\right),
$$

with $H(x)$ a Heavyside step function and $\alpha$ a normalization factor. A big advantage of choosing this parametrization over, e.g., a Drude-Lorentz model ${ }^{7}$ is that the final loss function will still be approximately local in terms of the parameters $a_{i}$. This is very beneficial for the stability of the fitting process.

The real part $\operatorname{Re}\left(\frac{1}{\varepsilon(E)}\right)$ can now be obtained by solving the KK equation analytically. We get

$$
\begin{aligned}
\operatorname{Re}\left(\frac{1}{\varepsilon(E)}\right) & =1-\frac{2}{\pi} \mathcal{P} \int_{0}^{\infty} \operatorname{Im}\left(\frac{-1}{\varepsilon\left(E^{\prime}\right)}\right) \frac{E^{\prime}}{E^{\prime 2}-E^{2}} d E^{\prime} \\
& =1-\frac{2}{\pi} \sum_{i} \mathcal{P} \int_{E_{i}}^{E_{i+1}} \alpha\left(a_{i} E^{\prime}+b_{i}\right) \frac{E^{\prime}}{E^{\prime 2}-E^{2}} d E^{\prime} \\
& =1-\alpha G(E),
\end{aligned}
$$

where $\mathcal{P}$ is the principal value and

$$
\begin{aligned}
G(E)= & \frac{2}{\pi} \sum_{i}\left[\frac{b_{i}}{2} \ln \left(\left|\frac{E_{i+1}^{2}-E^{2}}{E_{i}^{2}-E^{2}}\right|\right)\right. \\
& +a_{i}\left(E_{i+1}-E_{i}+\frac{E}{2}\right) \ln \left(\left|\frac{E_{i+1}-E}{E_{i}-E}\right|\right) \\
& \left.-\ln \left(\left|\frac{E_{i+1}+E}{E_{i}+E}\right|\right)\right]
\end{aligned}
$$

The scaling factor $\alpha$ can be determined by setting $E=0$

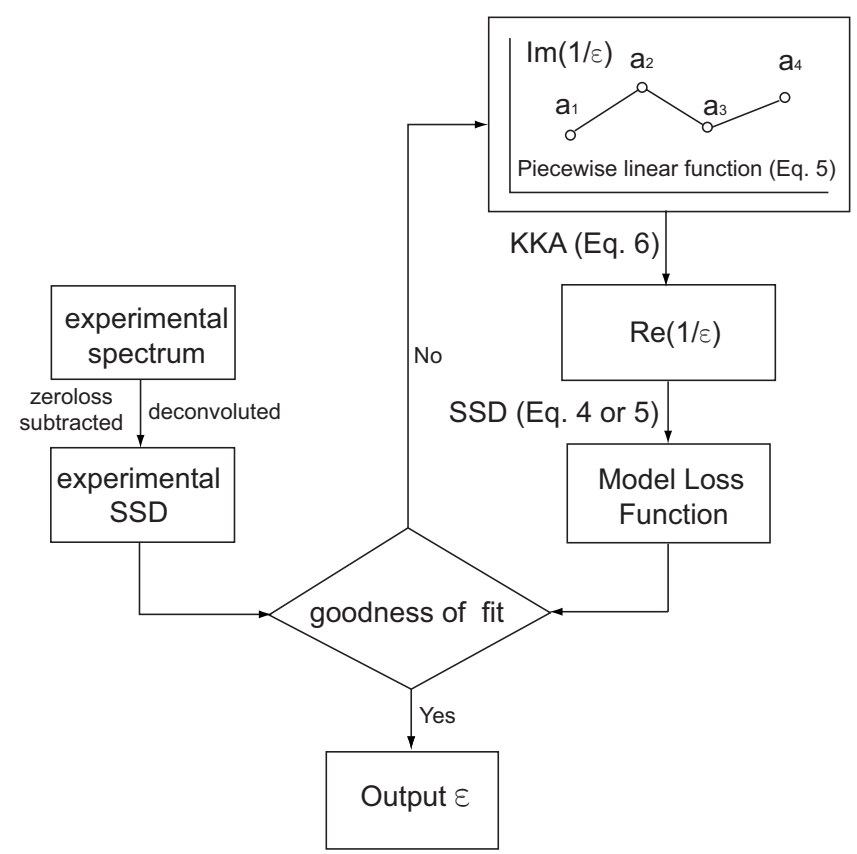

FIG. 2. Flowchart of the fitting procedure.

$$
\alpha=\frac{\left(1-\frac{1}{\varepsilon(0)}\right)}{G(0)} .
$$

Note that in case of incomplete knowledge of the loss function near $E=0$ (as usual in EELS), one can alternatively scale the result to $\operatorname{Re}\left(\frac{1}{\varepsilon(E)}\right)$ for an energy $E$ that is available from optical experiments.

This completes the model for the SSD. Making use of Eqs. (5)-(7) [or Eq. (4) for parallel illumination condition] we now have a parametric model that can be fitted to experimental data which includes retardation effects and is valid for bulk isotropic materials at low scattering angles. The result of a maximum likelihood fit with an experiment will be estimates of $a_{i}$ which can be easily transformed into $\varepsilon_{1}, \varepsilon_{2}$ or $n, \kappa$. The whole procedure is schematically displayed in Fig. 2.

\section{EXPERIMENT AND RESULTS}

\section{A. Bulk materials}

To test the proposed method, we first apply it to two bulklike samples. A first spectrum is obtained from bulk diamond on a JEOL 3000F equipped with a Gatan 2000 imaging filter. A second spectrum of bulk $\mathrm{SrTiO}_{3}$ is taken from the EELS database $^{16}$ and was obtained by B. Rahmati on a VG HB501UX dedicated STEM equipped with cold field emission gun and a GATAN ENFINA spectrometer. The samples are prepared with ion milling making them electron transparent. Strictly speaking they are therefore no longer bulk samples but rather thin films. The thickness is however still much larger than the mean free path for inelastic scattering which makes them behave as close as possible to a bulk material. 

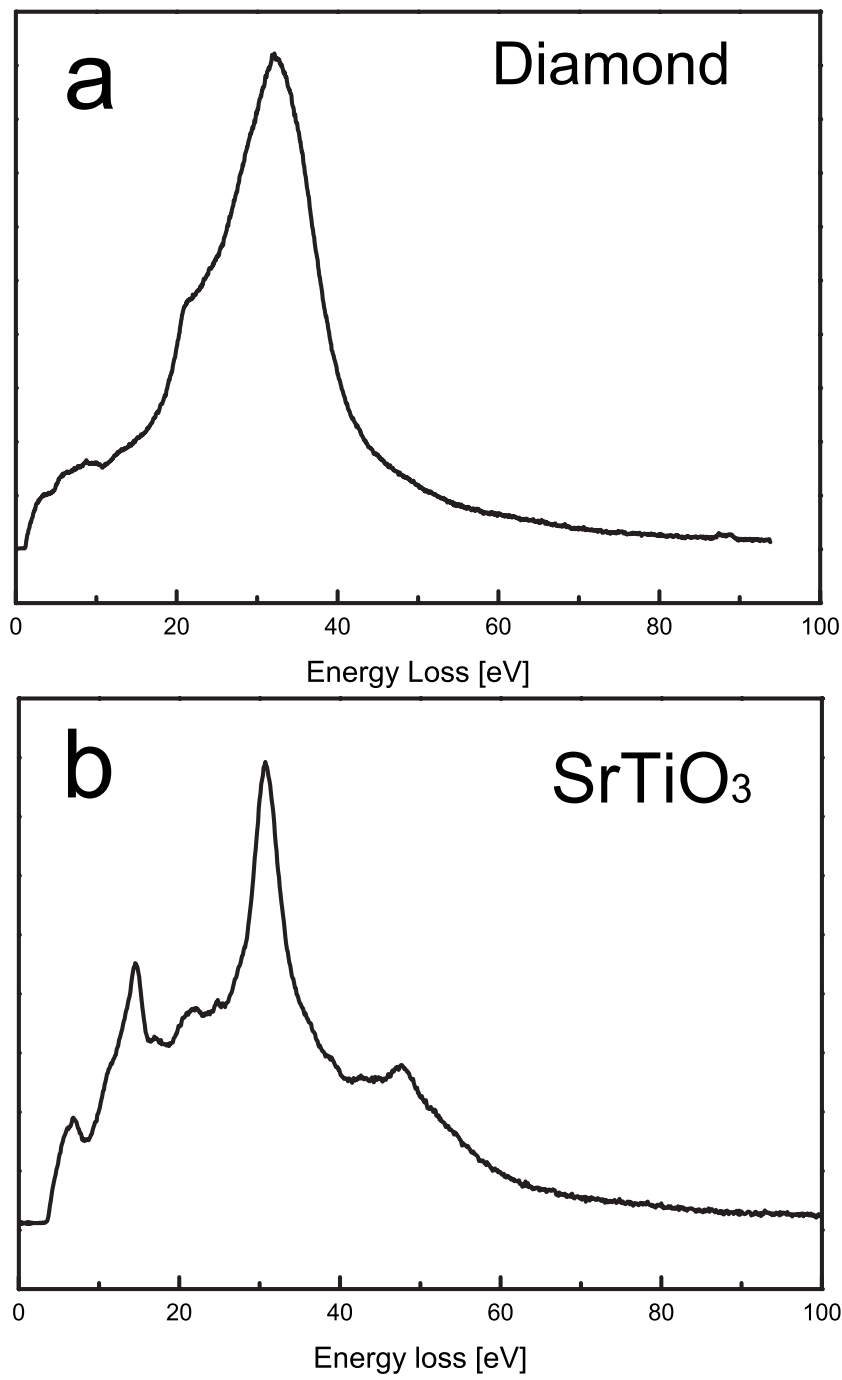

FIG. 3. Low-loss EELS spectra of bulk diamond (a) and $\mathrm{SrTiO}_{3}$ (b).

Both spectra are ZLP subtracted by local power law fitting and Fourier log deconvoluted. The result is shown in Fig. 3. These spectra serve now as the input for the method described in the previous section which is implemented in the EELSMODEL program. ${ }^{17,18}$ To show the importance of the relativistic correction, two different models are tried, one in which the relativistic effects are neglected by setting $\gamma=1$ and another one where we keep the relativistic effect. The results after fitting are shown in Figs. 4 and 5 together with optical data obtained from Palik ${ }^{19}$ for comparison. Scaling was done using low optical frequencies $(0.2 \mathrm{eV}$ for diamond, $0.4 \mathrm{eV}$ for $\mathrm{SrTiO}_{3}$ ) rather than static dielectric constants in view of the missing loss data for very low frequencies.

\section{B. Nanoparticles}

In view of the final goal of obtaining response functions from nano-objects, it would seem logical to apply the presented model on low-loss spectra obtained from, e.g., nanoparticles. In Fig. 6 we show low-loss EELS spectra for diamond nanoparticles with different diameters. The
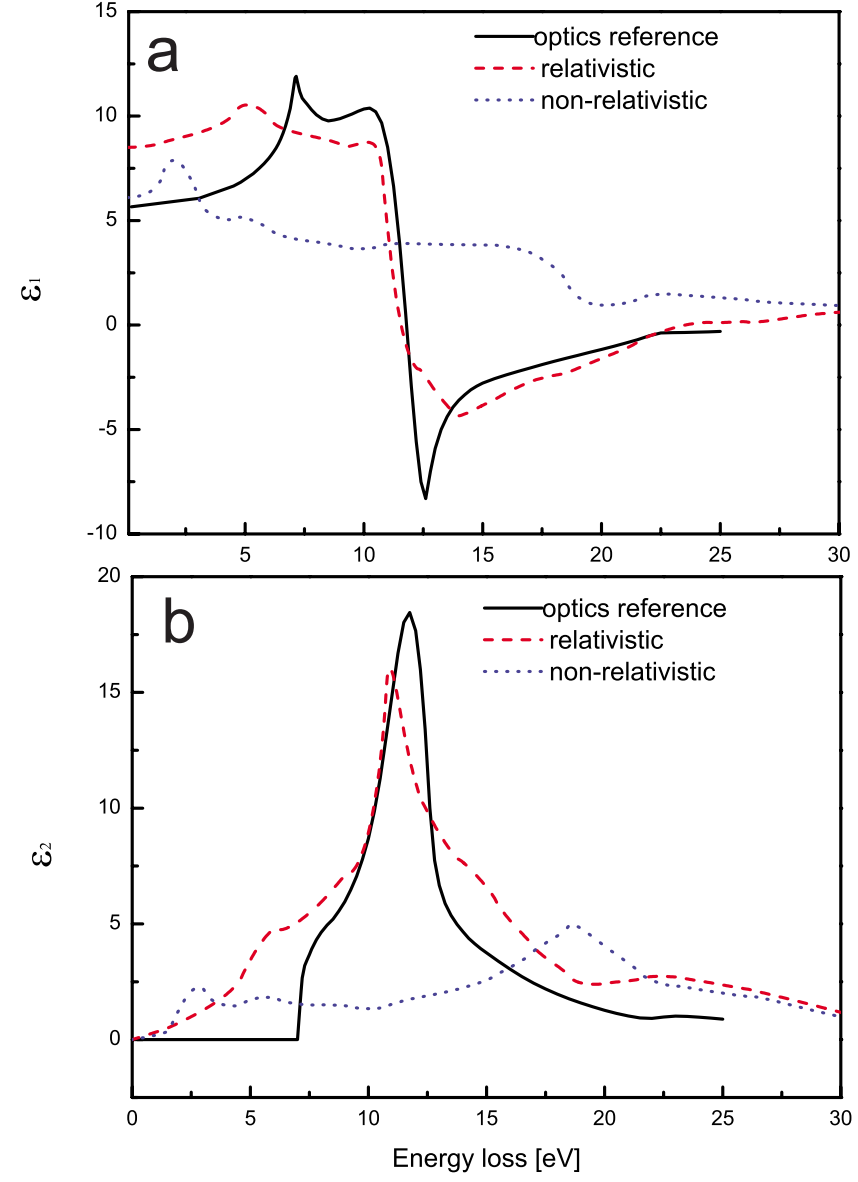

FIG. 4. (Color online) The real (a) and imaginary part (b) of the estimated dielectric function for bulk diamond obtained by modelbased fitting including retardation effects (broken red line) and without those effects (blue dotted line) compared to optical reference data from Palik (Ref. 19) (black solid line).

nanoparticles are obtained from detonation diamond which has been washed in acid to remove any amorphous carbon. This process is described in detail in Ref. 20 and leaves clean nanoparticles of diamond with sizes between 5 and $20 \mathrm{~nm}$. EELS spectra were obtained on a JEOL $3000 \mathrm{~F}$ operating at $300 \mathrm{kV}$ in STEM mode with a probe convergence angle of $4.4 \mathrm{mrad}$ and a spectrometer collection angle of $6.7 \mathrm{mrad}$. The energy resolution is estimated to be $\sim 1 \mathrm{eV}$.

Two peaks can be observed in each spectrum located at 32 and $22 \mathrm{eV}$, which originate, respectively, from a bulk and surface plasmon contribution. The ratio between the surface and bulk plasmon peak increases as the particle size decreases. Especially for the $5 \mathrm{~nm}$ particle, the surface plasmon peak is even more intense as the bulk plasmon peak which illustrates that the surface contribution becomes dominant.

Straightforward application of Eq. (2) is therefore no longer allowed since these were derived only for bulk samples. If we however would use a loss function including the effect of surfaces and retardation, we would eventually obtain the dielectric function for bulk diamond which is of little interest in this case since it no longer reflects the specific response of the nanoparticles. 


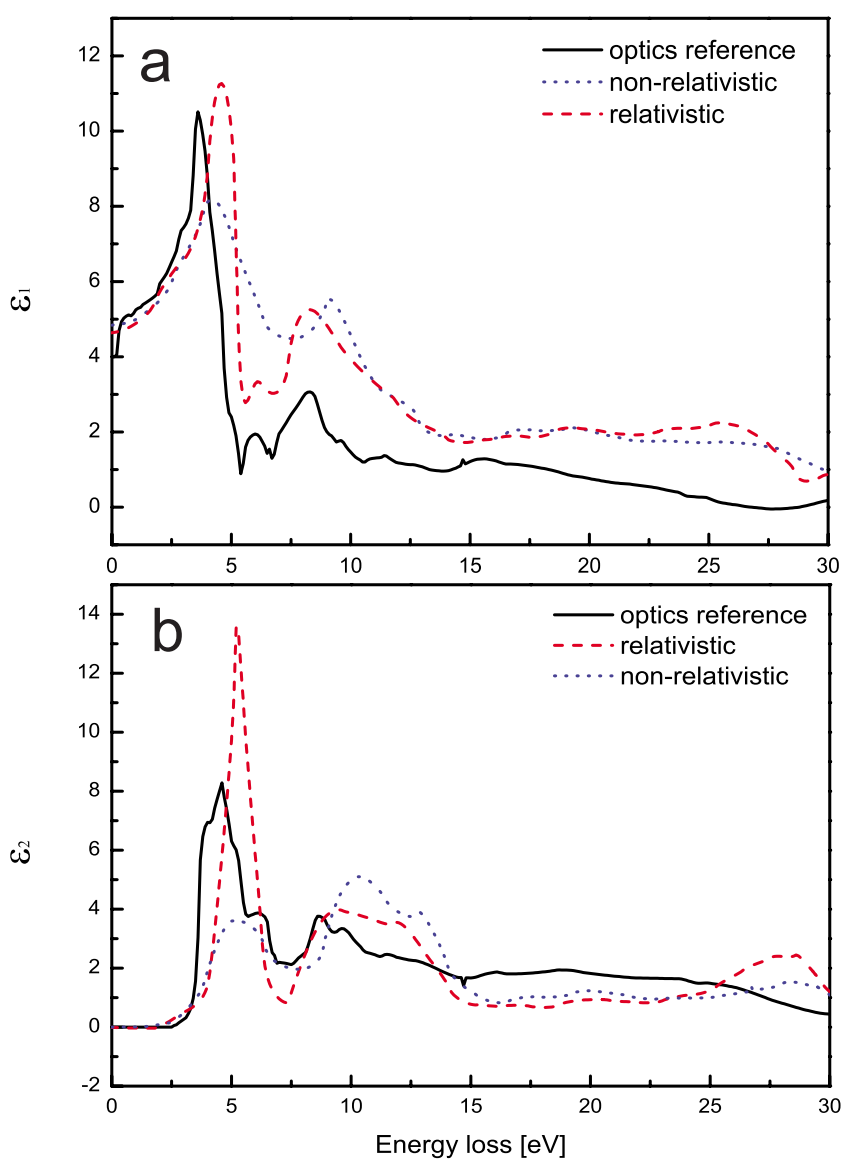

FIG. 5. (Color online) The real (a) and imaginary part (b) of the estimated dielectric function for bulk $\mathrm{SrTiO}_{3}$ obtained by modelbased fitting including retardation effects (broken red line) and without those effects (blue dotted line) compared to optical reference data from Palik (Ref. 19) (black solid line).

Ideally we would need a generalization of the retardation effects for the response in inhomogeneous media which would enable us to properly describe relativistic effects for particles of any shape. Looking at analytical formulas derived for specific shapes like spheres ${ }^{12,21}$ and thin foils $9,10,13$ it is clear that such a generalization is not possible as witnessed by the excessively complicated formulas describing the effects of bulk and surface effects and their retardation effect.

\section{DISCUSSION}

The application of the model to bulklike samples showed a rather good agreement with optical data especially when relativistic effects are taken into account. Diamond can be seen as a worst case for retardation in view of the high refractive index of $n=2.4$ (at $0.2 \mathrm{eV}$ ) (Ref. 19) as compared to $\mathrm{SrTiO}_{3}$ with $n=2.2$ (at $0.4 \mathrm{eV}$ ). ${ }^{19}$ This difference is clearly observed in the fact that for $\mathrm{SrTiO}_{3}$ the result without retardation correction is still reasonable while for diamond the correction becomes indispensable. This makes this model approach a viable alternative for optical methods with the advantage of collecting a very wide range of energies in one

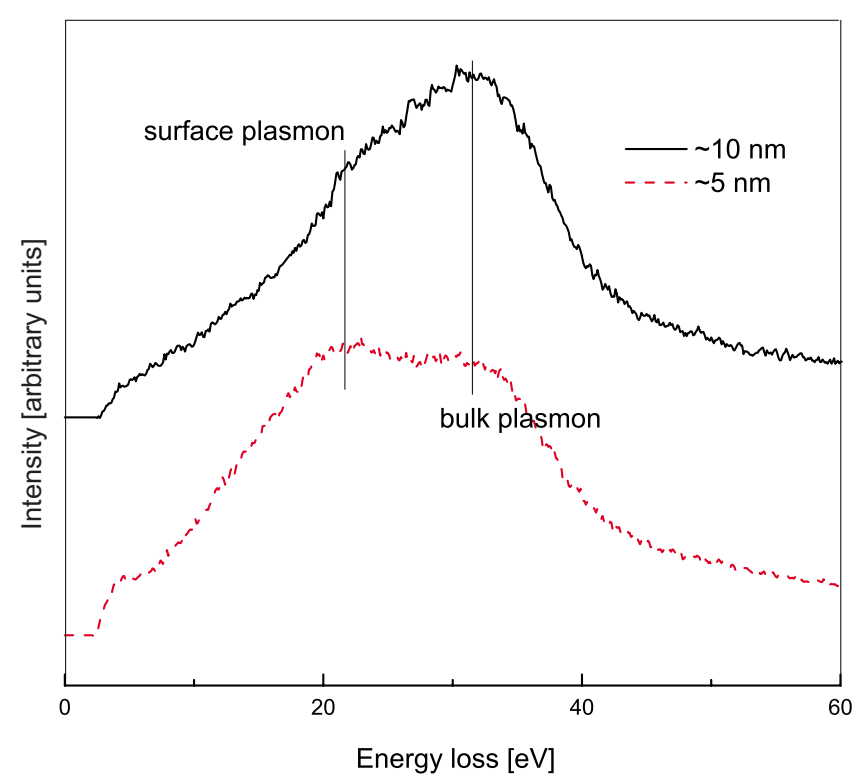

FIG. 6. (Color online) VEEL spectra of diamond particles with different sizes. The black solid line is for particles around $10 \mathrm{~nm}$ and the red broken line is for particles around $5 \mathrm{~nm}$

experiment with a reasonable spatial resolution. A source of inaccuracies in the obtained dielectric function is the lack of a good loss function for low energies in EELS $(<1 \mathrm{eV})$. This is mainly due to background subtraction and limited energy resolution. The effect of this on the obtained dielectric function is strongest for low energies because of the preferential sampling of the loss function near $E^{\prime}$ in Eq. (7). This can make the calibration of $\varepsilon(0)$ problematic in some cases.

A straightforward extension to nanoparticles was shown to be problematic because of the occurrence of surface excitations and the different way the retardation effects emerge in the bulk and surface contributions. This limit was to be expected because the loss as measured in EELS is determined by two elements:

(i) The boundary conditions: if one assumes a continuum model for the dielectric response, the loss function is completely determined by the shape and the environment of a given nanoparticle.

(ii) The dielectric function of the material: at extremely small dimension, the continuum approach to the dielectric function fails, and quantum mechanical effects start to play a role.

In comparison, the boundary conditions are by far the most dominant effect in realistically sized nanoparticles (containing easily more than a few thousand atoms). Although the size effect on the dielectric function is very interesting to measure, one can only do this when properly taking into account these boundary effects. Ideally we would model these boundary effects, but many obstacles arise.

(i) The exact shape of the nanoparticle is generally unknown.

(ii) The response of a system of nanoparticles depends in a sensitive way on the coupling to neighboring particles (position, shape, and material).

(iii) Even for a known distribution of all but the simplest shapes, analytic expressions for the loss function are not 
available and numerical simulations are needed.

If we can solve these issues for a given configuration of nanoparticles we eventually could obtain the dielectric function corrected for finite-size effects. We would like to stress however, that in many cases of technological interest, one is more interested in understanding which plasmonic modes exist and how coupling between different particles works. For this purpose it is much more interesting to look directly at the loss function rather than trying to convert it into a socalled dielectric function. The obvious failure of doing this (and neglecting the boundary conditions) was shown in Fig. 6 when applying our model to diamond nanoparticles. By working directly with the loss function, one can link to concepts such as the optical density of states as proposed by De Abajo. ${ }^{12}$ Unfortunately even the electron energy loss function is not a unique identifier of the sample at a given location because retardation and surface effects still depend on the speed of the probe electrons. Moreover there are delocalization effects which also depend on the acceleration voltage. Going to lower acceleration voltages could work but even at $80 \mathrm{kV}$ electrons still have a speed of $v \approx 0.5 \mathrm{c}$.

These arguments put a clear limit to the application of the presented technique. Nevertheless, the method still is able to give reliable dielectric information over a wide range of energies for sample dimensions where surface effects can be neglected.

\section{CONCLUSION}

Model-based quantification of low-loss STEM EELS has been applied to determine the dielectric function of bulklike materials. Diamond and $\mathrm{SrTiO}_{3}$ have been analyzed and show a good agreement with optical reference data if retardation effects are included. The method is included in EELSMODEL (Ref. 17) which is freely available to the community. In comparison to previous methods relying on multiple experimental spectra, this method has the advantage of only requiring one spectrum in a standard EELS setup.

Extending this method to nanoparticles proves to be difficult because retardation effects can not be transformed out if surface and bulk excitations occur together. Moreover we argue that the response of nanoparticles is governed to a large extent by their boundary conditions and measuring dielectric functions might not be appropriate.

\section{ACKNOWLEDGMENT}

All authors would also like to acknowledge the financial support from the European Union under the Framework 6 program, under a contract for an Integrated Infrastructure Initiative, Reference No. 026019 ESTEEM. J.V. is grateful to the Fund-for-Scientific-Research-Flanders under Contract number G.0147.06. Behnaz Rahmati is acknowledged for providing the $\mathrm{SrTiO}_{3}$ spectrum through the EELS database.

\footnotetext{
*Corresponding author. FAX: +3232653257; liang.zhang@ua.ac.be

${ }^{1}$ C. Kittel, Introduction to Solid State Physics, 8th ed. (Wiley, New York, 2004).

${ }^{2}$ J. A. Kittl et al., Microelectron. Eng. 86, 1789 (2009).

${ }^{3}$ F. J. García de Abajo, N. Zabala, A. Rivacoba, A. G. PattantyusAbraham, M. O. Wolf, and P. M. Echenique, Phys. Rev. Lett. 91, 143902 (2003).

${ }^{4}$ L. Zhang, R. Erni, J. Verbeeck, and G. Van Tendeloo, Phys. Rev. B 77, 195119 (2008).

${ }^{5}$ L. Gu, V. Srot, W. Sigle, C. Koch, P. van Aken, F. Scholz, S. B. Thapa, C. Kirchner, M. Jetter, and M. Rühle, Phys. Rev. B 75, 195214 (2007).

${ }^{6}$ M. Stöger-Pollach, Micron 39, 1092 (2008).

${ }^{7}$ R. F. Egerton, Electron Energy Loss Spectroscopy in the Electron Microscope, 2nd ed. (Plenum Press, New York, 1996).

${ }^{8}$ J. Verbeeck and G. Bertoni, Ultramicroscopy 109, 1343 (2009).

${ }^{9}$ E. Kröger, Z. Phys. 235, 403 (1970).
}

${ }^{10}$ R. Erni and N. D. Browning, Ultramicroscopy 108, 84 (2008).

${ }^{11}$ R. H. Ritchie and H. B. Eldridge, Phys. Rev. 126, 1935 (1962).

${ }^{12}$ F. J. García de Abajo, arXiv:0903.1669, Rev. Mod. Phys. (to be published).

${ }^{13}$ M. Stöger-Pollach and P. Schattschneider, Ultramicroscopy 107, 1178 (2007).

${ }^{14}$ M. Stöger-Pollach, Proceeding of MC2009 1, 113 (Verlag der Technischen Universitát Graz, Austria 2009).

${ }^{15}$ H. Kohl, Ultramicroscopy 16, 265 (1985).

${ }^{16}$ http://www.cemes.fr/eelsdb/.

${ }^{17} \mathrm{http}: / / \mathrm{www}$. eelsmodel.ua.ac.be/.

${ }^{18}$ J. Verbeeck and S. Van Aert, Ultramicroscopy 101, 207 (2004).

${ }^{19}$ E. D. Palik, Handbook of Optical Constants of Solids (Academic, New York, 1985).

${ }^{20}$ S. Turner, O. I. Lebedev, O. Shenderova, I. I. Vlasov, J. Verbeeck, and G. Van Tendeloo, Adv. Funct. Mater. 19, 2116 (2009).

${ }^{21}$ R. A. Ferrell, Phys. Rev. 111, 1214 (1958). 